\title{
Resolution and enhancement in nanoantenna-based fluorescence microscopy
}

\author{
Hadi Eghlidi, Kwang Geol Lee, Xue-Wen Chen, Stephan Götzinger, and Vahid Sandoghdar \\ Laboratory of Physical Chemistry and optETH, \\ ETH Zurich, CH-8093 Zurich, Switzerland
}

\begin{abstract}
Single gold nanoparticles can act as nanoantennas for enhancing the fluorescence of emitters in their near fields. Here we present experimental and theoretical studies of scanning antenna-based fluorescence microscopy as a function of the diameter of the gold nanoparticle. We examine the interplay between fluorescence enhancement and spatial resolution and discuss the requirements for deciphering single molecules in a dense sample. Resolutions better than $20 \mathrm{~nm}$ and fluorescence enhancement up to 30 times are demonstrated experimentally. By accounting for the tip shaft and the sample interface in finite-difference time-domain calculations, we explain why the measured fluorescence enhancements are higher in the presence of an interface than the values predicted for a homogeneous environment.
\end{abstract}


Since its realization in 1984 [1, 2], Scanning Near-field Optical Microscopy (SNOM) has demonstrated spatial resolution beyond the diffraction limit in several different contexts and configurations. Some of the most impressive reports of resolution have been made in the so-called apertureless SNOM mode where the intensity of a diffraction-limited illumination is enhanced in a small nanoscopic region very close to a sharp metallic tip [3, 4, 5, 6, 7, 8]. Despite its success in nonlinear [8] and interferometric infrared [9] near-field imaging, apertureless fluorescence SNOM has not become a routine tool in the laboratory. Moreover, theoretical modeling of the optical properties of tips made of real metals has posed numerous challenges so that quantitative agreements between theory and experiment have been missing. Several years ago, we proposed to use single spherical gold nanoparticles (GNP) attached to dielectric tips as well-defined and reproducible probes for apertureless SNOM [10]. Since then it has been shown that a GNP can act as a nanoantenna to enhance the fluorescence of a single molecule (SM) in its near field [11, 12, 13, 14]. The simplicity of this experimental arrangement has made it possible to compare the experimental findings with theoretical predictions.

As the GNP becomes smaller, the region to which its near-field intensity is confined shrinks, suggesting the possibility of achieving higher spatial resolutions. However, to benefit from this strong confinement, the molecule-GNP separation also has to be made smaller and this might in turn cause fluorescence quenching [11, 13, 15]. Viewed from a different perspective, smaller GNP antennas are expected to become less effective because the ratio of the scattering and absorption cross sections decreases with decreasing GNP diameter [16]. In this Letter, we examine the intricate interplay between excitation enhancement, quenching, and resolution as a function of the GNP diameter.

Our samples consisted of terrylene molecules embedded in ultrathin crystalline films of p-terphenyl (pT) spin coated on carefully-cleaned glass cover slides [12, 13, 15, 17]. In these films, terrylene molecules are aligned with their transition dipole moments at about $15^{\circ}$ to the normal to the plane of the sample. We excited the molecules by light from a solid-state laser at a wavelength of $\lambda=532 \mathrm{~nm}$ through a high numerical aperture microscope objective $($ N.A. = 1.4). As shown in Figure 1, the excitation laser beam was p-polarized to generate a strong $z$-component upon total internal reflection at the pT-air interface. Fluorescence from individual molecules, which was peaked at $\lambda=580 \mathrm{~nm}$, was collected with the same objective and directed either to a sensitive charge-coupled device (CCD) or to a single photon 
counting avalanche photodiode (APD). The fluorescence signal of a single molecule was then monitored while a GNP was laterally raster scanned across it at a constant gap $g$ from the pT-air interface. The details of tip preparation, tip-sample distance stabilization, as well as excitation and detection schemes can be found in Refs. [10, 12, 13, 15].

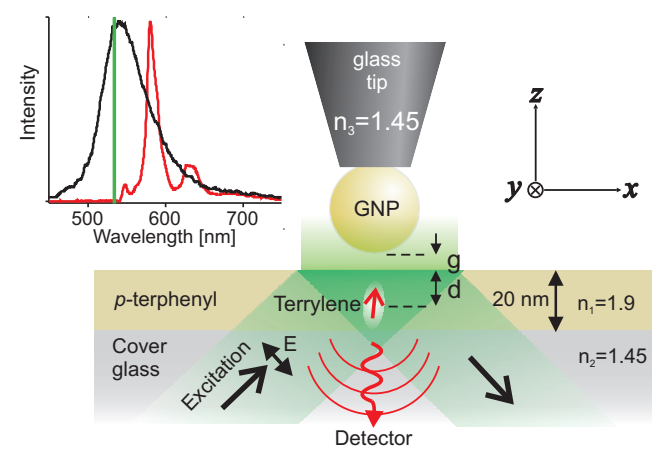

FIG. 1: Sketch of the experimental setup. Terrylene molecules are embedded in a thin crystalline $p$-terphenyl film and are illuminated under total internal reflection. The same objective collects fluorescence of individual molecules while a single gold nanosphere attached to a glass fiber tip is scanned across. Parameters $d$ and $g$ denote the depth of the molecule under the sample surface and the GNP-sample separation, respectively. Refractive indices $\mathrm{n}_{1}, \mathrm{n}_{2}$, and $\mathrm{n}_{3}$ show the values used for the theoretical calculations. The inset shows a plasmon spectrum of a GNP (black), the fluorescence spectrum of a single terrylene molecule (red), and the excitation laser line (green).

Figure 2a shows a $200 \mathrm{~nm}$ x $200 \mathrm{~nm}$ raster scan fluorescence image of a single terrylene molecule under a GNP with a diameter of $100 \mathrm{~nm}$. In Figure 2 $\mathrm{b}$, we plot a cross section from this image, reporting a full width at half-maximum $W=41 \mathrm{~nm}$ and a fluorescence enhancement $\Phi=8$. An electron micrograph (SEM) of the probe is displayed in the inset. To determine $\Phi$ for each SM, we carefully measured the sample fluorescence background from a region without the molecule and subtracted it from the fluorescence signals of the molecule in the presence and absence of the GNP. Furthermore, we accounted for the autofluorescence of each GNP. Figures $2 \mathrm{c}-\mathrm{h}$ present some examples of the results from experiments with GNPs that had diameters 80,60 , and $40 \mathrm{~nm}$. Figures $2 \mathrm{k}$,d report $\Phi$ as large as 30 while Figures $2 \mathrm{~g}, \mathrm{~h}$ show that $W$ can be as narrow as $18 \mathrm{~nm}$. Interestingly, $\Phi=16$ for a GNP with a diameter of $40 \mathrm{~nm}$, which is even larger than the value obtained in Figures $2 \mathrm{a}$, b for a GNP that is $100 \mathrm{~nm}$ in diameter.

To identify a size-dependent trend, we performed experiments with many antenna probes and SMs. Instead of reporting average values, we present the outcome of individual measurements for $\Phi$ and $W$ by the black symbols in Figures 3 c and d, respectively. For convenience, 

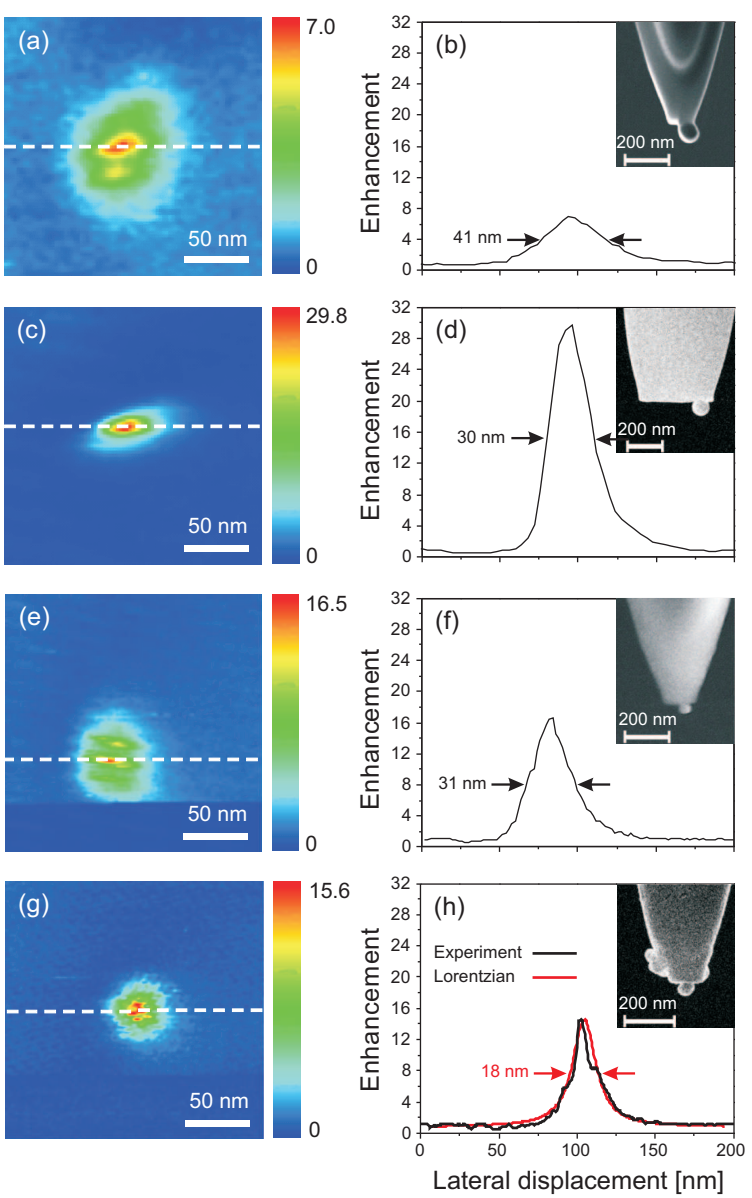

FIG. 2: Near-field fluorescence images of single molecules obtained using gold nanospheres with diameters of $100 \mathrm{~nm}$ (a), $80 \mathrm{~nm}$ (c), $60 \mathrm{~nm}$ (e), and $40 \mathrm{~nm}(\mathrm{~g})$. The enhancement is encoded in false colors. The images were recorded at a scan speed of $40 \mathrm{~nm} / \mathrm{s}$ with a pixel size of $2 \mathrm{~nm}$. The squeezed shape of the image in (c) is caused by a stage drift. b, d, f, h) Cross sections of the scan images on the left-hand side. The red curve in (h) displays a Lorentzian fit. The insets show SEM images of the gold nanospheres attached to the fiber tips.

we have labeled the lowest and highest values of $W$ for the data set of each particle size by the upward and downward pointing triangles. As expected, in most cases a low $W$ is correlated with a high $\Phi$ and vice versa. To examine the origin of the substantial spread in our findings, we now present the results of finite-difference time-domain (FDTD) calculations with convolutional perfectly matched layer absorbing boundary condition [18]. To minimize the computation time, we used the body of revolution FDTD method (bor-FDTD) whenever applicable [20]. In particular, we made the approximation that the molecular dipole moments are perpendicular to the pT film to benefit from the bor-FDTD calculations. The great advantage of this numerical approach over a simple analytical model [12, 19] is that it allows us to take into account the tip shaft, pT thin film, underlying glass substrate, as 
well as the evanescent excitation.

In the weak excitation regime, $\Phi$ is the product of the enhancement in the excitation intensity, modification of the quantum efficiency (QE), and change in the collection efficiency due to the presence of the GNP [12]. To find the first factor, we propagated a radially polarized Gaussian beam incident at an angle of 45 degrees in the bor-FDTD calculations to mimic a p-polarized illumination used in the experiment (see Figure 1). We then calculated the electric field intensity at different locations inside the $\mathrm{pT}$ film in the presence and absence of the antenna probe. Figure 3 a displays some examples of the outcome for $g=5 \mathrm{~nm}$ and different values of $d$ as a function of the GNP diameter. To evaluate the QE of the emitter for a given configuration, we computed the power radiated by the molecule into the far field as well as the total power dissipated (i.e. radiatively and nonradiatively) by the molecule and determined the ratio of the former to the latter. The red and blue curves in Figure $3 \mathrm{~b}$ show the calculated radiative and nonradiative decay rates normalized to the spontaneous emission rate of the molecule in the absence of the nanoantenna. The collection efficiency was calculated as the ratio of the radiated power into the substrate within a cone angle of 135 degrees to the total radiated power.

The solid curves in Figure 3r. summarize the results of computations for $\Phi$, indicating that the spread in $\Phi$ can stem from small variations of the distance $d$ between the molecule and the pT-air interface (see Figure 1). However, since the tip-sample distance in SNOM is not stabilized to an absolute value [21], it is also possible that the fluctuations are caused by small changes in $g$. An extended study of the various parameters such as $d, g$, and the refractive indices of the film and the substrate go beyond the scope of this paper and will be discussed in a forthcoming publication [22].

A sensitive dependence of $\Phi$ on the molecule-GNP separation is also expected from an analytical model of a molecule close to a GNP embedded in a homogeneous medium [12, 19]. However, the outcome of such a simple analysis, shown by the blue dashed curve in Figure 3 c for a molecule-GNP separation of $8 \mathrm{~nm}$ (equivalent to $g+d$ ), does not explain the observed fluorescence enhancements as large as 30 times [23]. As we had anticipated in our previous work [12], a quantitative understanding of $\Phi$ can only be obtained if one takes into account the sample-air interface. One of the effects of the interface is to lengthen the radiative lifetime of the excited state for a molecule with a dipole oriented normal to the interface [24]. For terrylene, which embeds in pT films at an angle of about $15^{\circ}$, this amounts to $4-5$ fold 
reduction of spontaneous emission rate compared to its bulk value. Thus, the quantum efficiency of these molecules is reduced from about 95\% to 79\% [25]. Calculations reveal that the onset of quenching is delayed by the interface so that for an axially-oriented molecule placed at $d=2 \mathrm{~nm}, g=2 \mathrm{~nm}$, the quantum efficiency is as high as $30 \%$ as compared to $15 \%$ for a GNP-molecule separation of $4 \mathrm{~nm}$ in a homogeneous environment with a refractive index of 1.35 [22]. Furthermore, the presence of the interface assists the antenna-induced enhancement of the excitation intensity and therefore $\Phi$.

The data in Figure 3r. show that fluorescence enhancement reaches an optimum for GNP diameters around $80 \mathrm{~nm}$ while $\Phi$ drops to values of the order of unity for GNP diameters of $40 \mathrm{~nm}$ and below. The solid curves in Figure $3 \mathrm{~d}$ display the expected trend of an improved resolution for smaller GNPs and shorter molecule-GNP separations. These data were obtained by calculating $\Phi$ for different lateral positions of the molecule and then assessing the FWHM of the fluorescence profile. Here, one has to keep in mind that in order to achieve higher resolutions with smaller GNPs, it is also imperative to reduce the molecule-GNP separation. The rise of $W$ observed in curves iv, v, and vi for small GNPs illustrates this point. The blue dashed curve shows that as opposed to the case of $\Phi$, the magnitude and trend of $W$ do not deviate by a great deal from the predictions of an analytical model for a homogeneous medium with a refractive index of 1.35 .

With our current samples, it was not possible to ensure GNP-molecule distances of only a few nanometers in a routine fashion. A theoretical scrutiny of this regime is also beyond our current work because of numerical difficulties as well as uncertainties in the knowledge of the dielectric functions of very small gold nanoparticles [16, 26]. Furthermore, we point out that in cases where quenching becomes important, it is a nontrivial task to determine $W$ because lateral cross sections could first show enhancement and then quenching, leading to complex line shapes [11, 13]. Indeed, in the $\Phi<1$ regime, quenching has to be used as a contrast mechanism for microscopy [27]. Finally, we point out that antenna-based microscopy is most efficient for imaging axial dipole moments as studied in this work, and that the competition between enhancement and quenching is very different for emitters with a transition dipole moment parallel to the substrate [15].

The ultimate task of any imaging technique is to describe unknown samples. It is, thus, important to examine the ability of antenna-based microscopy for resolving close-lying fluorophores in a dense surrounding. Let us consider the fluorescence signal of a single emitter 


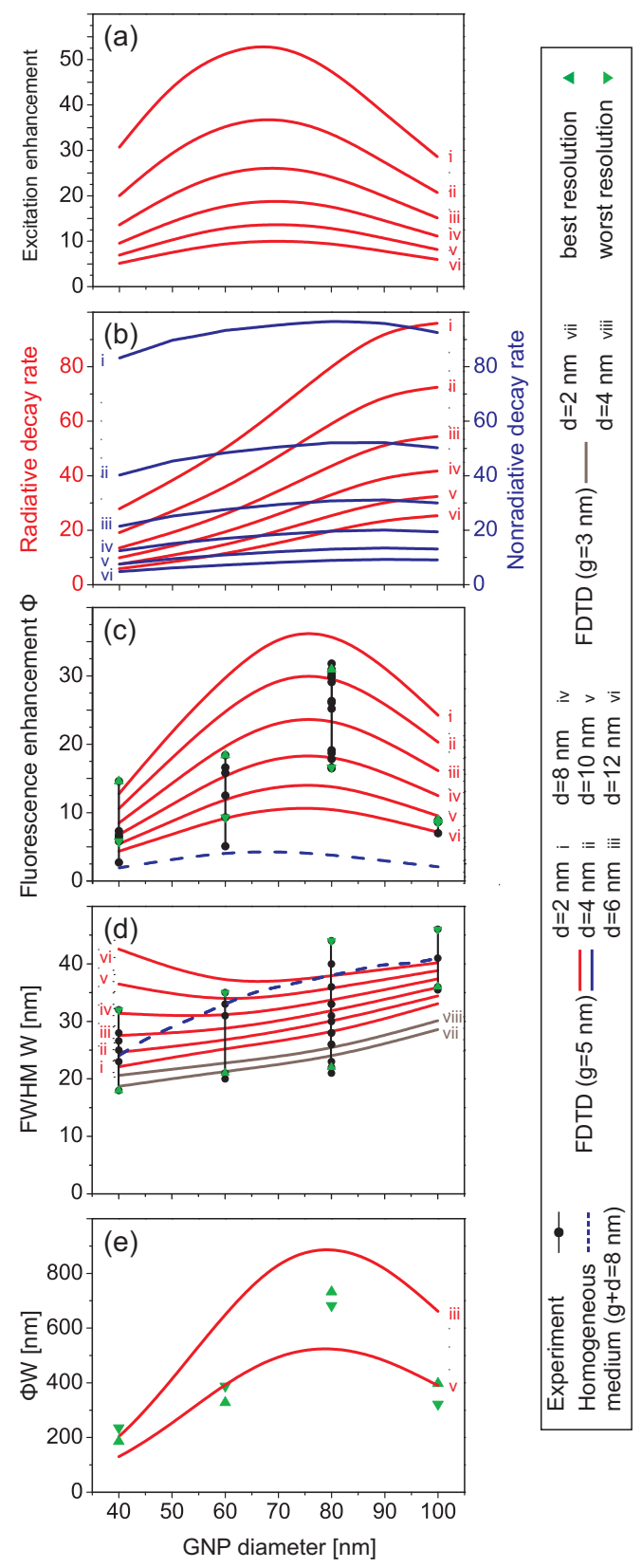

FIG. 3: a) Enhancement of the excitation intensity, b) radiative (red curves) and nonradiative (blue curves) decay rates normalized to the radiative decay rate in the absence of the GNP, c) fluorescence enhancement $\Phi, \mathrm{d}$ ) full width at half-maximum $W$, e) the product $W \Phi$. (a)-(e) are studied as a function of the GNP diameter. The black circles display the experimental measurements on single molecules. The solid curves show the results of the FDTD calculations for different molecule depths $d$ and tip-sample separations $g$ as specified in the legends; In (a)-(c) we employed bor-FDTD whereas in (d) we performed full 3D calculations to find the quantum efficiencies of laterally displaced molecules. The blue dashed curves depict the results of analytical calculations in a homogeneous medium of refractive index 1.35. The green triangular symbols pointing up and down denote the data corresponding to the highest and lowest observed resolutions, i.e. lowest and highest values of $W$, respectively. 
to be $S_{0}$ counts within the integration time. A uniform distribution of emitters at surface density $\delta$ molecules $/ \mathrm{nm}^{2}$ in a diffraction-limited detection spot yields a background signal of $S_{0} \delta \lambda^{2} /(2 N . A \text {. })^{2}$, whereas the signal from the enhanced region reads $S_{0} \Phi \delta W^{2}$. Here, $\lambda$ denotes the transition wavelength of the molecules. To decipher details at the resolution of $W$, the detected fluorescence signal should show a clear change as the antenna is scanned across an area of the order of $W^{2}$. The resulting ratio of the signal to the shot noise of the background becomes $3 W^{2} \Phi \sqrt{S_{0} \delta} / \lambda$ if one assumes a numerical aperture of $N . A .=1.5$. Therefore, if we neglect detector noise, achieving a signal-to-noise ratio (SNR) of 1 requires $\Phi W \gtrsim \lambda /\left(3 W \sqrt{S_{0} \delta}\right)$. Setting $\delta=1 / W^{2}$, provides a direct guideline $\Phi W \gtrsim \lambda / 3 \sqrt{S_{0}}$ for resolving single molecules spaced by $W$.

The symbols in Figure 3e display the experimental results of $\Phi W$ corresponding to the highest and lowest observed $W$ values reported in Figure $3 \mathrm{~d}$, and the solid curves show the theoretical values for two examples of molecular depth $d$. We find that the detection SNR is maximized when the GNP has a diameter of about $80 \mathrm{~nm}$ and the attainable resolution is in the 20-30 nm range (see Figure 3d). Although the experimental data for $\Phi W$ mirrors the same trend, their accuracy is not sufficient for a quantitative agreement with the theoretical predictions of the dependence on $d$. To assess the potential of antenna-based microscopy for ultrahigh resolution fluorescence imaging, we consider a realistic signal of $S_{0} \approx 1000-$ 10000 counts per second from a single fluorescent molecule at ambient condition and $\lambda=$ $600 \mathrm{~nm}$, yielding $\lambda / 3 \sqrt{S_{0}} \approx 7-2 \mathrm{~nm}$ after an integration time of one second. Thus, the data in Figures $3 \mathrm{~d}$ and 3 , show that it should be readily possible to resolve neighboring molecules with spacings below $20 \mathrm{~nm}$ even without the need for any fluorescence background suppression method [28, 29] although such techniques improve the SNR further.

In conclusion, we have shown that single gold nanoparticles serve as versatile and welldefined optical nanoantennas for high-resolution near-field fluorescence microscopy. By varying the particle size, we investigated the performance of this technique with respect to the overall attainable fluorescence enhancement and spatial resolution; we expect our findings to provide also a general trend for other optical nanoanntennas [30]. The reported experimental and theoretical data are in very good agreement and emphasize the near-field sensitivity of antenna-based microscopy to the exact position of the fluorophore. Furthermore, rigorous numerical calculations have shown that the dielectric-air interface imposed by the sample is responsible for providing particularly high excitation enhancements and low quenching 
as compared to a molecule-antenna assembly embedded in a homogeneous medium. Our analysis shows that single emitters can be detected at a resolution beyond $20 \mathrm{~nm}$ even in dense ensembles. By using ellipsoidal nanoparticles [20], it might be possible to push the resolution in fluorescence microscopy to the molecular scale.

\section{Acknowledgements}

We thank Mario Agio for fruitful discussion and Alois Renn for experimental help. This work was supported by ETH Zurich and the Swiss National Science Foundation (SNF).

[1] D. Pohl, W. Denk, and M. Lanz, Appl. Phys. Lett. 44, 651 (1984).

[2] A. Lewis, M. Isaacson, A. Harootunian, and A. Muray, Ultramic. 13, 227 (1984).

[3] F. Zenhausern, M. P. O’Boyle, and H. K. Wickramasinghe, Appl. Phys. Lett. 65, 1623 (1994).

[4] P. Gleyzes, A. C. Boccara, and R. Bachelot, Ultramicroscopy 57, 318 (1995).

[5] S. Kawata and Y. Inouye, Ultramicroscopy 57, 313 (1995).

[6] F. Zenhausern, Y. Martin, and H. K. Wickramasinghe, Science 269, 1083 (1995).

[7] E. J. Sanchez, L. Novotny, and X. S. Xie, Phys. Rev. Lett. 82, 4014 (1999).

[8] A. Hartschuh, Angew. Chem. Int. Ed. 47, 8178 (2008).

[9] F. Keilmann and R. Hillenbrand, Phil. Trans. Roy. Soc. Lond. A 362, 787 (2004).

[10] T. Kalkbrenner, M. Ramstein, J. Mlynek, and V. Sandoghdar, J. Micros. 202, 72 (2001).

[11] P. Anger, P. Bharadwaj, and L. Novotny, Phys. Rev. Lett. 96, 113002 (2006).

[12] S. Kühn, U. Håkanson, L. Rogobete, and V. Sandoghdar, Phys. Rev. Lett. 97, 017402 (2006).

[13] S. Kühn, G. Mori, M. Agio, and V. Sandoghdar, Mol. Phys. 106, 893 (2008).

[14] C. Höppener and L. Novotny, Nano Lett. 8, 642 (2008).

[15] S. Kühn and V. Sandoghdar, Appl. Phys. B 84, 211 (2006).

[16] U. Kreibig and M. Vollmer, Optical Properties of Metal Clusters (Springer Berlin, 1995).

[17] R. J. Pfab, J. Zimmermann, C. Hettich, I. Gerhardt, A. Renn, and V. Sandoghdar, Chem. Phys. Lett. 387, 490 (2004).

[18] A. Taflove and S. Hagness, Computational electrodynamics: the finite difference time-domain method (3rd edition, Artech house, 2005). 
[19] P. C. Das and A. Puri, Phys. Rev. B 65, 155416 (2002).

[20] A. Mohammadi, V. Sandoghdar, and M. Agio, New J. Phys. 10, 105015 (2008).

[21] K. Karrai and I. Tiemann, Phys. Rev. B 62, 13174 (2000).

[22] X. Chen, M. Agio, and V. Sandoghdar (in preparation).

[23] Treatment of optical spectra at interfaces is a nontrivial task, but many phenomena can be approximated by assuming a homogeneous medium with an effective refractive index. Here, we set $n=1.35$ as in Ref. [12], where the surrounding index was determined by fitting the GNP plasmon spectrum in that experiment.

[24] B. C. Buchler, T. Kalkbrenner, C. Hettich, and V. Sandoghdar, Phys. Rev. Lett. 95, 063003 (2005).

[25] For dipole moments perfectly perpendicular to the interface, a quantum efficiency of 0.95 is reduced to 0.67 . Since our bor-FDTD calculations of the decay rates in Fig. 3b assume such molecules, the results of the fluorescence enhancement are slightly overestimated.

[26] P. Stoller, V. Jacobsen, and V. Sandoghdar, Opt. Lett. 31, 2474 (2006).

[27] E. Yoskovitz, D. Oron, I. Shweky, and U. Banin, J. Phys. Chem. C 112, 1630616311 (2008).

[28] C. Xie, C. Mu, J. R. Cox, and J. M. Gerton, Appl. Phys. Lett. 89, 143117 (2006).

[29] C. Höppener, R. Beams, and L. Novotny, Nano Lett. 9, 903 (2009).

[30] T. H. Taminiau, R. J. Moerland, F. B. Segerink, L. Kuipers, and N. F. van Hulst, Nano Lett. 7, 28 (2007). 